ISBN 978-81-933894-1-6

International Conference on Studies in Disaster Management, Civil and Architectural Engineering

(SDMCAE-17)

Kyoto (Japan) April 18-19, 2017

\title{
Planning of Taking Parks as Temporary Emergency Shelters
}

\author{
Jieh-Jiuh Wang \\ Department of Architecture, Ming Chuan University, Taiwan
}

\begin{abstract}
The urban area is a major space where population, industrial and economic activities concentrate. Once the earthquakes attack the urban areas, it would undoubtedly result in large amount of victims. Where and how to accommodate those victims are big issues and should be prepared in advance. This study aims at earthquake disaster, discussed the way to convert the urban park into emergency shelter park. It intends to start from outlining the guidelines for planning, preparing and establishing shelters as well as the evaluation of suitability. The influence of hazard potentials are considered to help the reasonable evaluation of planners on the suitability of emergency shelter and improve the current configuration for shelter area, including location selection, spatial planning, facilities preparedness, and management system respectively.
\end{abstract}

Keywords: emergency shelter, urban park, earthquake, urban disaster management, emergency shelter pa

\section{Preface}

As the population in urbanised areas increases, the number and size of parks decreases, and the concentration of buildings intensifies, rapid urban growth and land acquisition become increasingly difficult; the cause for this is social and economic growth. If an urban area is affected by a disaster, such as an earthquake, the losses of life and property can be considerable. However, existing emergency shelters and regular practice can mitigate the effects of disasters and inefficient rescue efforts. Parks satisfy people's need for recreation while serving as emergency shelters and rendezvous points for emergency response units in case of disasters. Emergency shelter parks (ESP) are designed mainly as a response to earthquakes, with a primary function being to provide shelter for earthquake victims. Few studies have emphasised the theories and principles of ESPs. This study aimed to develop a planning and evaluation model for selecting parks and converting them into ESPs.

\section{Epistemological and Technical Perspectives toward Emergency Shelter Park}

\subsection{Epistemological Perspective}

Disasters cause not only loss of life and property but also psychological impacts on society and individuals. A psychological crisis is a psychologically unbalanced condition that can occur when an individual or group cannot manage the implications of being affected by a sudden major event concerning public safety.[10] A psychological crisis is a process from which most people recover; however, certain people are affected by psychological disorders, and the worst results can be permanent suicidal tendencies.[6] Thus, the preparation for and response to victims' disaster psychology should be considered by governments and planners. The use of ESPs involves the initial response during and after a disaster, which is necessary for managing "disaster shock" or "disaster syndrome" in victims (i.e., scenarios in which people experience temporary numbness and confusion and begin to panic and try to escape; this might be accompanied by a lack of companions that experienced the disaster, high levels of sensitivity to existing difficulties, and the perception of being isolated and without support.[8] After 2 to 24 months of a disaster, people generally realise that their lives are not returning to a normal state and that they should expect no more aid. Strong emotions such as disappointment, anger, and 
resentment emerge; this is the beginning of the stage of disillusionment. In the stage of extended recovery, emotional problems may become stronger and more severe if post-disaster reconstruction works do not progress as smoothly as expected, and psychological crises may escalate. However, for disasters that cause severe and violent impacts in a short period of time, such as earthquakes, "mass panic" is inevitable and should be considered.

Evacuation behaviours influence the effectiveness of the planning of shelter facilities for different disaster types. Several studies have examined certain disaster types and evacuation behaviours.[2][5][21][24] In addition, numerous studies on earthquakes have been published.[7]Error! Reference source not found.[15][16][23] These studies are the foundations for shelter facilities and special care must be given to disadvantaged groups during disasters. Disadvantaged groups are not independent of each other, but they have multiple attributes (i.e., a person may be disadvantaged both naturally and socially). Reactions after disasters and levels of resilience vary among groups.

In the event of an earthquake, it is vital to provide emergency and temporary shelters as well as rescue posts throughout the chaos period and until the following shelter period. An ESP is a park or open space that comprises various disaster management functions in the case of an urban disaster event and is designed for versatile purposes and tasks. It is a space that functions as both a typical urban park and a shelter and that is used in various disaster stages to manage the impacts of disasters.[9] The tasks and functions ascribed to ESPs by researchers of various fields vary depending on the characteristics of specific disaster stages (e.g., initial, emergency, and recovery stages [9]).

\subsection{Technical Perspective}

The Office for the Coordination of Humanitarian Affairs (UN/OCHA) (2010) proposed 10 guiding principles concerning shelter, settlement, and recovery. The principles are consistent with those stated in the Global Facility for Disaster Reduction and Recovery (GFDRR) (2010) and include strategy, reconstruction, community, disaster risk reduction, coordination, development, relocation, stakeholders, assessment, and sustainability. Mifune (1995) proposed four principles for evaluating and selecting a shelter.[1] Sheltering occurs in four stages [4]: (1) emergency shelter; (2) temporary shelter; (3) temporary housing; and (4) permanent housing. ESPs are involved mainly during the emergency shelter stage and have the primary functions of providing emergency shelters for the distressed, a base for rescue activities and recovery efforts, and a space for disaster management education during times of normal operation. ESPs have at least 8 functions: evacuation; shelter; information exchange and communication; firefighting support, rescue, medical service and emergency response efforts; temporary victim support; assistance in epidemic control and sanitation; reconstruction support; and transportation support.[17]

The ESP system of Japan comprises the following types: regional disaster prevention points, regional evacuation sites, evacuation roads, temporary shelters, neighbouring disaster prevention points, and green buffer zones for isolating petrochemical zones. The planning process comprises planning stages (9 steps) and operational management ( 3 aspects). Facility planning involves 10 levels: square and route, vegetation, water resource facilities, toilets for emergency use, communication equipment, power supply equipment, lighting facilities, storage and warehouses, operation centres, and other facilities.[17][18]

The Construction and Planning Agency (CPA), Ministry of the Interior, Taiwan, indicated in 1997 that parks must have sanitation and security functions and serve as shelter sites in the case of air raids and emergency evacuations.[13] The Department of Urban Development, Taipei City Government proposed guidelines according to which at least $40 \%$ of the available space of plazas and green spaces are used for emergency shelter, and evacuation is considered in the design of paths and entrances, as well as in the installation of fire-blocking green belts, emergency shelter, evacuation indication systems, emergency lighting, water supplies, and additional disaster prevention facilities.[3]

A shelter is defined as a habitable covered space providing a secure and healthy environment with privacy and dignity for those residing in it.[25] For the consideration of privacy and dignity, the number of people 
accommodated in a shelter should be limited. The distance of evacuation is estimated according to the accessibility by foot. The criteria differ among countries; in the US, the shelter distance is defined as 0.5 miles; in Taiwan, there are three types of sheltering area, neighbourhood sheltering (500-700 m), regional sheltering (1,500-1,800 m), and city-wide sheltering (the entire city; [26]).

The effective operations of urban disaster management depend heavily on the accessibility of a transportation system. "Disaster evacuation involves immediate and rapid removal of persons from the threat area.[21] Wickramaratne et al. (2011) indicated that "successful evacuation is the ultimate decisive factor of the whole warning process since an incomplete evacuation could cause thousands of lives to be lost."[22] In Taiwan, disaster relief roads are defined in the following manner: those $8 \mathrm{~m}$ or more in width are for emergency evacuation; those $15 \mathrm{~m}$ or more in width are for firefighting, emergency response, and rescue; and those $20 \mathrm{~m}$ or more in width are for material supplies, external communications, support, and emergency shelter, if necessary.[19] The outward access conditions of a shelter determine the disaster management functions. The CPA [14] proposed five types of emergency road:

- Emergency access roads, at least $20 \mathrm{~m}$, are used to ensure barrier-free access to the affected areas. To connect to other districts, and to crucial rescue bases. Traffic control may be necessary for rescue efforts.

- Rescue roads, at least $15 \mathrm{~m}$, are used to transport rescue supplies, equipment, and personnel. They form a complete rescue road network with the emergency access roads.

- Emergency evacuation roads are those used by affected people to access emergency shelters. Every street block must connect at least two emergency evacuation roads to prevent hampering the rescue efforts if the only access road is blocked.

- Firefighting roads are sandwiched between incombustible buildings and provide sufficient width for vehicle passage and space for the manoeuvres of firefighting vehicles.

- Alternative roads are those not included as firefighting roads or emergency evacuation roads. These roads, according to the hazardous locations in a city, are alternative routes for evacuation and rescue efforts in a disastrous event.

The Green Space and Disaster Prevention Association (GSDPA) proposed two types of ESP-related facility: an "ESP facility" and a "typical park facility that can be used for disaster management," including landscaped, resting, recreational, exercising, cultural and educational, and public and management facilities as well as others.[27]

Five types of program configuration for shelters have been suggested: the command and management zone (including the command post and registration), accommodation zone (including a single men's quarter, single women's quarter, family quarter, and special care quarter), material management and catering zone (including supply distribution area and storage area), medical service (including prayer rooms), and a zone for other supporting services (including reception and bathrooms). The accommodation zone is the most crucial.

The preparation for a shelter includes disaster management bases and facilities. The soundness of disaster management bases are highly related to the environmental conditions (e.g., square, internal routes, entrance, firefree buffer trees and established park instalments), and facilities refers to the establishment or improvement of facilities (e.g., emergency operation base as well as water storage, emergency, indication, communication, sheltering, and firefighting facilities;[20]). Peng and Lin (2003) suggested that the timeline of a disaster and the response actions should be examined separately.[11] For the evacuation stage, sufficient and safe sheltering space should be provided; for the rescue stage, the key is to provide initial medical service and material supplies for the victims, and therefore, attention should be paid to the storage and use of materials; and for the accommodation stage, the sufficient supply of materials and equipment must be ensured to allow the victims enough time to relocate to long-term housing. 


\section{Evaluation Process and Framework}

\subsection{Definitions and Settings}

An ESP provides recreational and relaxing functions and fulfils emergency response requirements; therefore, ESPs must meet at least four basic conditions: (1) a sufficient sheltering area; (2) adequate accessibility; (3) adequate connections to neighbouring facilities; and (4) a program configuration that meets the demands of emergency scenarios.

ESPs can be divided into four types: temporary emergency shelters, neighbourhood ESPs, regional ESPs, and city-wide ESPs.

- Immediately after a major earthquake, people evacuate to nearby temporary shelters. These are open spaces, such as small parks and green spaces at street corners, with an area of at least $500 \mathrm{~m}^{2}$ and a service radius of $300-500 \mathrm{~m}$;

- Neighbourhood ESPs are for short-term accommodation and require a space of approximately 1-3 ha with a sheltering area of 2,000-6,000 $\mathrm{m}^{2}$, which is sufficient for accommodating $500-1,500$ people. These spaces require a service radius of $600 \mathrm{~m} \mathrm{[3]} \mathrm{and} \mathrm{must} \mathrm{provide} \mathrm{accommodation} \mathrm{for} \mathrm{3-14} \mathrm{days.} \mathrm{The}$ adjacent roads are emergency evacuation roads at least $8 \mathrm{~m}$ in width.[11][19]

- When medium or long-term accommodation is required, people are relocated to regional or city-wide ESPs. These must be 3-8 ha in size and able to accommodate 1,500-2,000 people. These parks must connect directly to at least one emergency access that is at least $20 \mathrm{~m}$ wide or to a rescue road at least 15 $\mathrm{m}$ in width. The service radius is $2 \mathrm{~km}$.

- City-wide ESPs must be at least 8 ha in size, with a usable shelter space of at least $8,000 \mathrm{~m}^{2}$, and accommodate 2,000 people. In addition to having a large capacity, these parks must serve several key roles, such as accommodating a command post, logistics support centre, and recovery base. Therefore, these parks must connect directly to at least one emergency access that is at least $20 \mathrm{~m}$ wide or to a rescue road at least $15 \mathrm{~m}$ in width.

\subsection{Development of Planning and Evaluation Process}

In the context of shelter response to natural disasters, shelter is a process in addition to a product.[25] This study developed a set of evaluation, planning, and operation processes that simplify the entire planning process. The process comprises: location selection, program configuration, facility preparation, and a management system. Location selection is a required process, whereas the other three processes are based on particular needs.

\subsubsection{Location Selection}

An investigation of particular disaster histories, existing functions, the location, the natural environment, the evacuation area, and additional necessary information is required. Knowledge of the effective sizes of parks and the widths of connecting roads enables ensuring the functionality and accessibility of parks. The key point in selecting the location for an ESP is the evaluation of environmental hazard characteristics, which is a required evaluation; combining this evaluation with a park-environment-status survey enables evaluating the external environment and internal space of parks.

Large inflows of victims are expected during a disastrous event. It is necessary to monitor the population dynamics in various locations and apply the collected information in space management. Evaluating an ESP should entail determining the size and shape of the park, the usable shelter space, and access to installed facilities. Typically a park with a size exceeding 0.6 ha and of which at least $40 \%$ is usable shelter space qualifies as an ESP.

\subsubsection{Program Configuration}


The second stage involves zoning as well as estimating the sheltering space and capacity. Regarding zoning, the necessary facilities in an ESP comprise three types, administrational management, life-support, and water utility facilities, which are arranged depending on the environment of individual parks. The administrational management facilities are used to maintain the its functions and are staffed by personnel from public offices, as well as volunteers. The life-support facilities are provided to satisfy the basic needs for daily life. The water supply is the most crucial component of basic life needs. Regarding the estimation of sheltering space, once the information of an environmental survey is available and the established park instalments (buildings, vegetation, etc.) are deducted from the total park area, the effective sheltering space is calculated and the capacity is determined on the basis of a space requirement of $4 \mathrm{~m}^{2}$ per person.

\subsubsection{Facility Preparation}

Facility preparation involves estimating material demands and tent configurations. In estimating material demands, the number of facilities required for preparing an ESP is based on the ESP carrying capacity. These facilities include accommodation, water utility, sanitation, emergency response, power supply, lighting, communication, signs, as well as food resources and life-support supplies.

Regarding the tent configuration, the number and location of required tents and how they are erected are estimated on the basis of the capacity. Walkways between tents and the distance from vegetation must be considered. Because most families have six or fewer members, tents for six people $(2 \times 2.7 \mathrm{~m})$ are used to estimate capacity.

\subsubsection{Management System}

The management system of an ESP involves typical park management and ESP management. The park management department has the management responsibility, because parks are provided for recreation and relaxation during times of normal operation. Once an ESP is established in a time of disaster, an authorising centre must assume management. Therefore, a general management program is required particularly for the water supply as well as for building and waste management.

\section{Conclusions and Suggestions}

Because of climate change and the increasingly unpredictable risk of disasters, implementing adaptation strategies and disaster prevention measures has become crucial. The configuration of an ESP addresses the need for emergency shelter and the accommodation of victims of major earthquakes. The following valuable conclusions were reached:

- This ESP evaluation standard is a set of simple principles that consider the environmental conditions of a park and the population distribution in individual areas.

- The four stages of transforming a current park into ESP is proposed. This procedure enables selecting an appropriate site and systematically developing it into an ESP.

- The key points for disaster management include the autonomous management of people in a disaster area and the support from civilian volunteers in the case of a disaster.

- Regarding the preparation of materials, a meticulously designed and appropriate open-contract system should be established to prevent a situation in which stored materials perish and are unusable in the case of a disaster.

\section{References}

[1] A. Mifune, Regional urban disaster prevention development, Tokyo: Ohmsha Ltd.,1995 .

[2] C. A. Tai, Y. L. Lee and C. Y. Lin, "Urban disaster prevention shelter location and evacuation behavior analysis," Journal of Asian Architecture and Building Engineering, vol. 9 No. 1, pp. 215-220, 2010.

https://doi.org/10.3130/jaabe.9.215 
[3] C. F. Tsai, Urban Disaster Prevention Regulations and Strategies, Taipei: Architecture and Building Research Institute, Ministry of the Interior, 2002.

[4] D. Félix, J. M. Branco and A. Feio, "Temporary housing after disasters: A state of the art survey," Habitat International, vol. 40, pp. 136-141, 2013.

https://doi.org/10.1016/j.habitatint.2013.03.006

[5] E. D. Kuligowski, The Process of Human Behavior in Fires, U.S.: National Institute of Standards and Technology, U.S. Department of Commerce, 2009.

https://doi.org/10.6028/nist.tn.1632

[6] D. Long, "Concept, types, changes, and results of mental crisis," Youth Studies, vol. 12, pp. 42-45, 1998.

[7] E. L. Quarantelli. (1980). Evacuation behavior and problems: Findings and implications from the research literature, Available: http://udspace.udel.edu/handle/19716/1283 (accessed 17 January 2017).

[8] E. L. Quarantelli, "Panic behavior: Some empirical observations", in Human Response to Tall Buildings, ed. D. J. Conway, PA: Dowden Hutchinson and Ross Inc., pp. 335-350, 1977.

[9] E. Ishikawa, "Planning of disaster prevention park (in Japan)," Landscape Architecture, vol. 34, pp. 57-62, 2000.

[10] H. C. Chiu and R. Q. Liang, Stress and Psychological Crisis Intervention, Guangzhou: Jinan University Press, 2008.

[11] J. J. Wang, S. C. Chang and Y. Y. Yang, Park as Emergency Shelter in Hsinchu City, Hsinchu: Fire Bureau, Hsinchu City Government, 2012.

[12] K. H. Peng and F. T. Lin, Standard Operation Procedure of Post-Disaster Emergency Shelter, Taipei: Architecture and Building Research Institute, Ministry of The Interior, 2003.

[13] M. Kuo, "The new role of urban park in environmental changes," Open Space and Parks, vol. 2, pp. 6-15, 2000.

[14] National Taipei University of Technology (NTUT), Urban Road Transport Emergency Planning Manual, Taipei: Construction and Planning Agency, Ministry of the Interior, 2009.

[15] N. Dash and H.Gladwin, "Evacuation decision making and behavioral responses: Individual and household," Natural Hazards Reviews, vol. 8, No. 3, pp. 69-77, 2007.

https://doi.org/10.1061/(ASCE)1527-6988(2007)8:3(69)

[16] N. Yun and M. Hamada, "Evacuation behaviors in the 2011 Great East Japan Earthquake," Journal of Disaster Research, vol. 7, No. 7, pp. 458-467, 2012.

[17] Organization for Landscape and Urban Green Infrastructure (OLUGI) and Green Space and Disaster Prevention Association (GSDPA), Disaster Prevention Park Instruction Handbook, Tokyo: Environmental Communications Co. Ltd., 2000.

[18] Organization for Landscape and Urban Green Infrastructure (OLUGI), Disaster Prevention Park Instruction Handbook II, Tokyo: Environmental Communications Co. Ltd., 2005.

[19] S. J. Wang and P. C. Xin, "Using park as disaster prevention and shelter," in The $4^{\text {th }}$ Forum of Urban Planning, Tainan: Department of Urban Planning, National Cheng Kung University, 2000.

[20] S. T. Lin, "The research of the metropolis takes measures against natural disasters the park service detailing criterion," M.S. thesis, Chung Hua University, 2006.

[21] S. W. Durage, L. Kattan, S. C. Wirasinghe and J. Y. Ruwanpura, "Evacuation behaviour of households and drivers during a tornado - Analysis based on a stated preference survey in Calgary, Canada," Natural Hazards, vol. 71, pp. 1495-1517, 2014. https://doi.org/10.1007/s11069-013-0958-6

[22] S. Wickramaratne, J. Y. Ruwanpura and S. C. Wirasinghe, "Decision analysis for a tsunami detection system- Case study: Sri Lanka," Civil Engineering Environment System, vol. 28, No. 4, pp. 353-373, 2011. https://doi.org/10.1080/10286608.2011.637625 
[23] T. Miki, T. Fukushima and T. Hori, "An analysis of refuges and temporary housing on the South Hyogo Prefecture Earthquake", in proceedings of symposium on infrastructure planning in Japan, 4-5 September 1997, pp. 129-136.

[24] T. W. Schmidlin, B. O. Hammer, Y. Ono and P. S. King, "Tornado shelter-seeking behaviour and tornado shelter options among mobile home residents in the United States," Natural Hazards, vol. 48, pp. 191-201, 2009.

https://doi.org/10.1007/s11069-008-9257-z

[25] UN/OCHA, Shelter after disaster: strategies for transitional settlement and reconstruction, 2010.

[26] Y. S. Chang, Urban Disaster Prevention and Planning, Taipei: Urban and Rural Development and Planning Agency, Taiwan Provincial Government, 1999.

[27] Y. Kimura, "Disaster prevention related facilities," Landscape Architecture, vol. 34, pp. 63-71, 2000. 